\title{
DESAFÍOS DE LA REGIONALIZACIÓN Y EL DESARROLLO ECONÓMICO EN PERÚ
}

\author{
REGIONALIZATION CHALLENGES AND ECONOMIC DEVELOPMENT \\ IN PERU
}

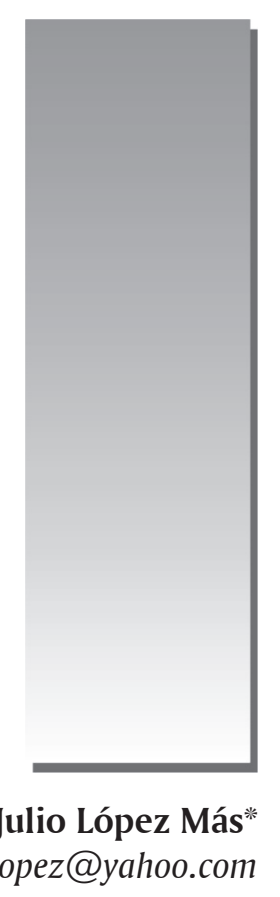

Johans Julio López Más*

johans_jlopez@yahoo.com

\begin{abstract}
RESUMEN
El proceso de regionalización debe proponerse la conformación de macrorregiones transversales. Esta nueva visión del desarrollo significa un reencuentro con las potencialidades del territorio, para reducir brechas economicas y desigualdades sociales. Las Juntas de Coordinación Interregional (Internor, Centro Sur, Amazónica, Macrosur) son agrupamientos voluntarios entre Gobiernos regionales. Estas lograron identificar un conjunto de proyectos articuladores (28 proyectos), que promoverían la intregración física y emprendimientos para el desarrollo económico local. Ademas, las JCI, en acción conjunta con las Agencias Regionales de Fomento de la Inversión Privada, generarían condiciones para conformar futuras regiones sostenibles. Para este fin, hay que hacer cambios en la política de inversión pública del MEF y realizar modificaciones en la normatividad de la descentralización, para crear unidades ejecutoras en las JCI, con agendas de desarrollo de corto y mediano plazo, unidades de planificación y programas de inversión multianuales adecuadamente financiados.
\end{abstract}

Palabras claves: Gobiernos regionales, Juntas de Coordinación Interregional, Agencias Regionales de Fomento de Inversión Privada, proyectos de inversión articuladores.

\begin{abstract}
The regionalization process, aim the formation of transverse macro-regions. This new vision of development means a reunion with the region's potential to reduce economic and social inequalities gaps. Interregional Coordination Meetings (ICM) (Internor, South Central, Amazon, Macrosur) are voluntary groupings among regional governments were able to identify a set of articulators projects (28 projects), which would promote physical integration and enterprises for local economic development. In addition, the ICM, in joint action with the Regional Development Agencies Private Investment, would generate future sustainable conditions to form regions. To this end, we must make changes to the Public Investment Policy of the Ministry of Economy and Finance (MEF), and also make changes in the regulations of decentralization to create execution units in ICM, with development agendas of short and medium term planning units and programs adequately funded multi-year investment.
\end{abstract}

Keywords: Regional Governments; Interregional Coordination Meetings; Regional Development Agencies Private Investment: Investment Projects articulators.

\footnotetext{
* Profesor principal de la Facultad de Ciencias Administrativas; docente investigador y ex director de Instituto de Investigaciones en Ciencias Administrativas; doctor en Ciencias Sociales. Este artículo es un resumen del Informe Final del Proyecto CON/CON 2010: Emprendimientos, Descentralización y Desarrollo Económico Local en Perú.
} 


\section{INTRODUCCIÓN}

El proceso de descentralización peruano, en la etapa actual, se inició en marzo de 2002. La reforma constitucional entiende la descentralización como un proceso a realizarse por etapas, de forma progresiva y ordenada, para asegurar la adecuada transferencia de responsabilidades y recursos a los Gobiernos regionales y locales. Se eligió las autoridades y se instalaron los Gobiernos regionales, sobre la base de los actuales departamentos y la Provincia Constitucional del Callao, a los que se les reconoce autonomía política, económica y administrativa para cumplir con sus competencias. Pero el proceso de descentralización debía continuar con la conformación de regiones a través de la unión de dos o más departamentos contiguos.

La Constitución delega a la voluntad ciudadana la decisión de la conformación de regiones, establece que mediante referéndum podrán integrarse dos o más circunscripciones departamentales contiguas para constituir una región. El mismo procedimiento para las provincias y distritos que, en su momento, decidan cambiar de circunscripción regional.

El texto constitucional delega en la ley la determinación de competencias y facultades adicionales, y de los incentivos especiales a las futuras regiones. Prevé, asimismo, que mientras dure el proceso de integración, dos o más Gobiernos regionales podrán crear mecanismos de coordinación entre sí. En este marco fueron creadas las Juntas de Coordinación Interregional y, por iniciativa del Gobierno anterior, se trató, de organizar regiones piloto (Áncash, Huánuco, Ucayali). (PRODES, 2011:125)

En conclusión, el modelo peruano de descentralización considera la conformación de regiones como unidades de desarrollo territorial y de gobierno descentralizado.

\section{PLANTEAMIENTO DEL PROBLEMA Y OBJETIVOS.}

El desarrollo económico local es el proceso de la dinámica económica, social y política de un área geográfica específica -dentro de las fronteras de una economía (país o nación)- resultante del comportamiento, acciones o interacciones de los agentes (económicos, políticos y sociales) que residen en el área geográfica, y que tiene la finalidad de incrementar sostenida y sosteniblemente el nivel y la calidad de vida de los habitantes de dicha area geografica, usando plena y eficientemente sus recursos humanos y no humanos (Tello, 2008:40).
El territorio es fundamento para la integración de políticas públicas, económicas, sociales, ambientales, institucionales. Es el lugar del pacto social de concertación, es el objetivo del planeamiento Descentralizado; debe ser unidad de planeamiento, presupuesto y gestión. (Dammert, SD-PCM, 2011: 13).

La presente investigación recoge evidencia empírica para identificar los mecanismos mediante los cuales los factores internos y externos de las regiones, así como las acciones e interacciones de los Gobiernos regionales, inciden en la promoción de inversiones públicas y privadas, en la integración económica interregional e intraregional, en beneficio de sus poblaciones.

En un Informe de Desarrollo Humano para el Perú, el PNUD reitera la necesidad crucial de lograr avances significativos en el cierre de brechas de acceso a los servicios públicos seleccionados, puesto que en muchas provincias del país los déficits de provisión de servicios básicos para el desarrollo humano (identidad, salud, educación, saneamiento y electrificación) alcanza el $60 \%$ o más. (PNUD, 2009: 17).

Consideramos a las Juntas de Coordinación Interregional como mecanismos de cooperación y coordinación entre Gobiernos regionales para facilitar la integración económica y la regionalización.

Las JCI, desde su creación entre 2004 y 2010, se han articulado en torno a proyectos, planes y programas, pero insuficientes como iniciativas de integración interregional. Trataremos de demostrar que lograr el funcionamiento adecuado y eficiente de las JCI, para fortalecer sus iniciativas de realización de obras y prestación conjunta de servicios, permite construir espacios (económicos y sociales) macroregionales e interdepartamentales.

Para un cabal contexto del problema, se analizan los aspectos teóricos y la evidencia empírica de los procesos de descentralización en países latinoamericanos, y se propone como un mecanismo del modelo de desarrollo sustentable de las JCI el concepto de proyecto de inversión estructurante o proyecto ancla.

\section{MARCO TEÓRICO E HIPÓTESIS}

Una evaluación comparada de las experiencias latinoamericanas, en materia de descentralización y promoción del desarrollo endógeno, devela 
una serie de derroteros comunes y aprendizajes que se pueden ordenar en hipótesis teóricas de intervención pública. Se presenta a continuación, de manera crítica, los planteamientos esbozados por Barrios ( PNUD/IDEA, 2012).

Hipótesis 1. Sobre las Macrorregiones territoriales de largo plazo preceptivamente organizadas desde el nivel nacional, no inmediatamente conectadas a una revisión de límites políticoadministrativos (Barrios, 2012: 32).

Se observa un callejón sin salida en las experiencias de Colombia, Ecuador y Perú, las que han confundido, y luego tratado de articular, la dimensión espacial con la territorial. La primera hipótesis sugiere que, desde el nivel nacional, se inicie un programa sostenido de inversión en nodos geoeconómicos por macrorregiones, que no dependan de fusión previa alguna de unidades político-administrativas, como los departamentos. Una posible configuración podría trabajar en torno a cuatro nodos alternativos a Lima, ubicados en el norte (Chiclayo/Piura/Trujillo), sur (Arequipa/ Cusco/Puno), centro (Huancayo) y una zona especial (Amazonía). Con este enfoque se han realizados interesantes estudios, tenemos la Estrategia de Regionalización de Macroconsult (2010) y la propuesta de Plan Nacional de Descentralización, PND 2012-2016 de la SD-PCM( diciembre 2011).

Las opciones de ser la base de unidades político-administrativas futuras no se descartan, pero se plantean para el largo plazo (veinte a treinta años). Un plazo largo es necesario, tanto para lograr los efectos espaciales de cohesión económica como para configurar una hipotética absorción de estos territorios en una unidad macroterritorial de nivel intermedio estatal. La necesidad de un largo plazo en la dimensión espacial se deduce de la lenta maduración de inversiones dirigidas al desarrollo económico. La creación de macrorregiones desde el nivel nacional implicará, sin embargo, la recuperación de mecanismos coercitivos de conformación de macrorregiones. Adicionalmente, se podría organizar por macroespacio una mínima estructura gerencial y sujetar administrativamente dichos espacios a un régimen dinámico de desconcentración, vale decir, uno donde el nivel nacional ejerza un control de legalidad y de mérito sobre las intervenciones de estas agencias de inversión, con su tecnoburocracia.

Esta primera hipótesis se basa en alentar preceptivamente desde el nivel nacional un "ma- duramiento espacial" de largo plazo en torno a nodos espaciales geoeconómicos, donde la consideración de una nueva configuración políticoadministrativa es accesoria y de muy largo plazo. Este es el camino "francés" de regionalización, y coincide en buen grado con propuestas que, en el mismo sentido, circulan actualmente en el debate peruano.

Esta política pública debe apostar por afectar sosteniblemente aquellos efectos del contrapeso espacial a Lima, que son crecientemente inmanejables, y que se derivan del modelo económico. Pero en los últimos años, Lima ha venido perdiendo peso demográfico relativo a favor de la constelación de urbes de más de cien mil habitantes. De hecho, desde la perspectiva espacial, la urbanización de otros nodos urbanos diferentes de Lima, ha implicado una desconcentración de facto en el país. Sin embargo, Lima sigue representando alrededor del $50 \%$ del producto económico del Perú. La urbe peruana que le sigue inmediatamente (Arequipa) tiene una alícuota nueve veces menor .

La regiones francesas logran su estructuración como entes territoriales con la reforma del 2003, por lo menos treinta años después de que dichas regiones se iniciaran como polos espaciales de desarrollo infraestructural.

Hipótesis 2. Política pública dirigida al equilibrio espacial macrorregional, en círculo virtuoso con la descentralización como medio de atacar la disparidad interterritorial (Barrios, 2012: 34).

Mientras la primera hipótesis de intervención estratégica propone separar espacio de territorio y apostar por un proceso largo y orgánico, que pueda eventualmente unirse con la división política administrativa, se entiende en esta segunda hipótesis, una serie de intervenciones públicas que permitan reducir los niveles de inequidad territorial en relación con el acceso a servicios básicos. Con este enfoque, no serían solo los cuatro nodos espaciales descritos en la primera hipótesis, los puntos de focalización, sino aquellas áreas de menor desarrollo. Se ha identificado a la sierra sur, la sierra norte, la selva alta y la Amazonía baja como las áreas más deprimidas del Perú, y con una densidad estatal muy baja (PNUD, 2009). La atenuación de las inequidades es uno de los temas que no resolvería la hipótesis 1 y corresponde, en buen grado, a políticas de descentralización y de desconcentración, de alianzas entre los tres niveles de Gobierno para actuar intradepartamen- 
talmente. Se ha planteado, incluso, considerar la provincia, y no el departamento, como la unidad básica de planificación y gestión para un desarrollo territorial exitoso.

Hipótesis 3. Reversión de las técnicas inequitativas de asignación fiscal. Tanto la hipótesis 1 como la hipótesis 2 presuponen que el Perú transforme su matriz fiscal territorial. El canon ha distorsionado el desarrollo armónico del sistema territorial. Su efecto es generalmente abrupto y volátil, razón por la cual no puede servir como fuente predecible de un sistema estable. Adicionalmente, como es usualmente voluminoso, impacta decisivamente en el desarrollo territorial del país. Las modalidades de asignación han agravado las inequidades horizontales y se debe rediseñar el sistema. La tercera hipótesis aboga, pues, por un pacto fiscal que reduzca ostensiblemente los efectos distorsionantes de la aplicación fluctuante de recursos, sustituyendo el principio de asignación en función de la ubicación geográfica de yacimientos de recursos naturales no renovables, en búsqueda de una asignación de cohesión territorial.

Con la lógica del canon actual, no solo se agravan las inequidades territoriales horizontales, sino que también se desalienta la fusión en regiones, amén de que el canon tiende a desarrollarse sin directa correlación con las tareas de desarrollo. Bolivia, Ecuador y Colombia, no han podido resolver este dilema.

Al mismo tiempo, en el Perú, los procesos de asignación fiscal deben ser correlativos con alteraciones de la carga competencial asignada a los entes territoriales. Para ello, debe diseñarse con claridad una técnica de distribución de cargas fiscales que aclare si financia las tareas del ente que las administra, o aquel que las legisla. Bajo las nuevas condiciones, se debería alentar la descentralización fiscal, con la ampliación del espacio tributario departamental sin esperar fusión de ellos en regiones. El análisis correcto de la descentralización requiere del manejo articulado de dos conceptos, autonomía y descentralización. La autonomía funciona en marco de una gestión descentralizada del desarrollo, con competencias y atribuciones, y recursos financieros, logísticos, humanos, necesarios para hacer eficiente y eficaz dicho proceso (PND, 2011:212).

Como señala acertadamente Efraín Gonzales, la descentralización fiscal y la desconcentración económica son procesos difíciles pero no impo- sibles. La descentralización fiscal, tributaria y del gasto tiene como objetivo mejorar la equidad territorial y humana (Gonzales, 2004:172).

Síntesis: Iniciativas de integración desde abajo y ausencia de plan orientador.

Luego de haber analizado las experiencias latinoamericanas más significativas en términos de descentralización (económica, administrativa, fiscal) y promoción del desarrollo territorial sostenible, identificamos como variables de investigación las relaciones de interrelación entre la gestión de las Juntas de Coordinación interregional, el fomento de la inversión pública articuladora en espacios territoriales descentralizados y su impacto en el desarrollo económico local.

El escenario del actual Gobierno 2011-2016 está marcado por la promoción de la inversión privada descentralizada, muy diversa desde grandes corporaciones nacionales y extranjeras, extractivas, mineras y petroleras; agroexportadoras; financieras, comerciales y de la industria de la construcción hasta iniciativas de emprendimientos regionales y locales de pequeños y medianos capitales, y de empresas asociativas agrarias, que coexisten con objetivos disímiles y acciones poco articuladas, muchas veces limitadas por la acción de las propias autoridades regionales y municipales.

No existe un marco legal para canalizar las iniciativas de integración regional. La Ley de Bases de la Descentralización y la Ley Orgánica de Gobiernos Regionales están desfasadas en relación con la realidad de las iniciativas nuevas, pues no cuentan con normas que faciliten estos espacios de articulación e integración. Si bien existen normas con relación a las JCI y las mancomunidades regionales, en parte sus funciones son similares. Y para lo que no existe marco normativo es para las regiones piloto.

El estudio de Macroconsult, realizado a pedido de la SD-PCM, titulado Propuesta Orientada a la Efectivización del Plan Nacional de Regionalización, señala que los incentivos para la creación de regiones son débiles (beneficios en el FONIPREL; prioridad y tramite preferencial para crédito internacional; endeudamiento interno por créditos a plazos superiores a 10 años; asignación del $50 \%$ de la recaudación de algunos impuestos internos nacionales, etc.), lo que no ayuda a crear un marco claro de funcionamientos de estos, ni contribuye a que la población y las autoridades regionales vean en ellos un escenario de beneficios atractivo que motive la integración (Macroconsult, 2010: 33). 
El actual momento del proceso de integración regional alerta sobre la necesidad de contar con un plan de regionalización, aunque de característica flexible, para que se perciba como una imposición a la población y a las autoridades descentralizadas; es decir, que se legitime a partir del debate y el consenso (PRODES, 2011: 135).

Pareciera que las condiciones del proceso de descentralización en la actualidad no tienen bases de consolidación institucionalidad y de gobernabilidad para avanzar una perspectiva de regionalización con un modelo cuasifederal. Para otros analistas, la única razón que podría justificar hoy la regionalización en nuestro país es el federalismo, o, mejor dicho, el federalismo aplicado a un Estado unitario, al que se le podría denominar como un cuasifederalismo o semifederalismo, como Canadá o España (Muñoz, 2013: 253-259).

La potencialidad de las JCI reside en ser una herramienta flexible para facilitar un tránsito gradual hacia las futuras regiones, en el proceso de formación de territorios económicos, aprovechando la existencia secular de dos grandes dinámicas; una longitudinal y otra transversal, lo que encaja con la conformación de grandes espacios macrorregionales (Santa Cruz, 2007:63 y 64).

Nuestra hipótesis de trabajo sostiene que la propuesta de conformar regiones mantiene vigencia, las macrorregiones territoriales de largo plazo, de carácter económico y político-administrativo. El fracaso del referéndum del 2005 y la falta de claridad en los procesos posteriores no le restan pertinencia a la integración y conformación de regiones, sino que obligan a revisar los enfoques, las estrategias, los procedimientos y las normas. Sin embargo, el proceso de regionalización no reposa solamente en las ventajas económicas comparativas, sino que además hay que reducir la disparidad interregional. En suma, una combinación de las hipótesis teóricas de intervención estratégica 1 y 2 discutidas en el marco teórico.

Las razones de fondo que sustentan la necesidad de formar regiones en el Perú son diversas: En lo político, es indispensable constituir núcleos de poder regional, con los recursos y capacidades suficientes, para contrarrestar el poder centralizado de la capital (Lima y Callao producen 50\% del PBI). Ricardo Vergara ha llamado la atención sobre la aceleración del proceso de urbanización, así se ha incrementado la población que reside en ciudades con más de 100 mil habitantes $(86.8 \%$ en
1961 y $58.4 \%$ en 2007), y estos sistemas urbanos pueden constituirse en una centralidad alternativa a Lima, y con impactos positivos sobre el mundo rural (Vergara, 2010:144-150). En lo económico, la integración regional amplía las posibilidades de las economías de escala y de la competitividad, generando impactos efectivos de carácter regional, con el procesamiento de recursos de sus territorios, con generación de empleos, ingresos y bienestar de sus poblaciones.

En lo territorial, siendo el Perú un país de territorios diferenciados, resulta necesario y favorable para el desarrollo otorgar a esos territorios una expresión política estable y democrática. En lo administrativo, la integración regional puede ser una de las vías para atenuar la fragmentación de las instituciones, de los presupuestos y de las capacidades de gestión en instancias de gobierno y territorios pequeños, enfrentando así la idea errónea de que descentralización es necesariamente atomización y dispersión.

Finalmente, la integración en regiones puede contribuir a mejorar la complementariedad y cooperación entre Gobiernos regionales y locales, superando la duplicidad y superposición, a través de diversas formas de asociatividad.

La presente investigación trata de demostrar que el fortalecimiento de la gestión de las Juntas de Coordinación Interregional favorece la articulación macrorregional, mediante la gestión de proyectos de inversión articuladores, con una perspectiva de regionalización de largo plazo y de desarrollo humano.

\section{MÉTODOS Y RESULTADOS.}

Las JCI constituyen un mecanismo de coordinación y cooperación interregional destinado a facilitar la integración regional. La Ley Orgánica de Gobiernos Regionales, Ley N² 29867, establece que a iniciativa de los Gobiernos regionales o del Consejo Nacional de Descentralización (Secretaría de Descentralización de la PCM), aquellos podrán establecer, de común acuerdo, Juntas de Coordinación Interregional (artículo $91^{\circ}$ ).

Por su parte, la Ley de Incentivos para la Integración y Conformación de regiones, Ley $\mathrm{N}^{\circ}$ 28274, establece que las juntas están integradas por dos o más Gobiernos regionales. Tienen por objetivo la gestión estratégica de integración para la conformación de regiones sostenidas y para la materialización de acuerdos de articulación ma- 
crorregional, mediante convenios de cooperación dirigidos a conducir los proyectos productivos y de servicios y alcanzar su integración para la conformación de regiones (artículo $3^{\circ}$ ).

Las Juntas de Coordinación Interregional (JCI) son vistas como promotoras de integración y coordinación regional, pero no con mucha claridad respecto al papel concreto que puedan desempeñar y cómo podrían ser potenciadas. Otros especialistas, más escépticos, indican que las limitaciones de su propia naturaleza hacen que las JCI nunca lleguen a ser instancias medulares para el desarrollo de las regiones (Muñoz, 2013:241).

Un aspecto de un enorme valor estratégico para una regionalización exitosa es la articulación y convergencia entre la dinámica intermedia o departamental y la dinámica propiamente local a nivel de las provincias. La articulación regionallocal puede y debe resolverse con un enfoque territorial que ubique los espacios o instituciones con mayor capacidad para operar como puentes o bisagras de esa conexión (PNUD 2009:19).

Sobre esos lineamientos de política, la Secretaría de Descentralización de la Presidencia del Consejo de Ministros está elaborando una propuesta de nuevo Plan de Descentralización y Regionalización, que está en proceso de revisión por el poder ejecutivo. (PCM-SD, 2012).

En la actualidad se carece de un instrumento metodológico que facilite la toma de decisiones en los procesos de priorización de proyectos de inversión, en ámbitos interregionales, y, en ese sentido, posibilitar su ejecución conjunta por los Gobiernos regionales integrantes de cada Junta de Coordinación Interregional. Para llenar este vacío la Secretaría de Descentralización de la PCM aprobó la Guía Metodológica para Priorización de Proyectos de Inversión y Articulación en Espacios Territoriales Descentralizados (noviembre de 2011). El responsable de este proyecto de investigación participó activamente en su condición de jefe de la Oficina de Inversiones de la SD-PCM.

Se define un proyecto estructurante al proyecto que articula las inversiones en un territorio, formando un grupo de proyectos y que genera sinergias; su beneficio e influencia es interdepartamental.

El planeamiento territorial y la inversión en los últimos años en América Latina para la priorización y ejecución de proyectos de inversiones en espacios territoriales de interrelación entre departamentos, regiones o estados federativos, que forman ejes nacionales integradores están aplicando la metodología multicriterio y la experiencia internacional da cuenta de múltiples beneficios: en Colombia, a través de la Dirección Nacional de Planeamiento; Brasil, mediante el Ministerio de Planeamiento, Presupuesto y Gestión; IIRSA, Iniciativa para la Integración de la Infraestructura Regional Suramérica, a través de MIDEPLAN de UNASUR.

Proyecto estructurante o proyecto-ancla. Los proyectos son agrupados en torno a un proyecto definido como proyecto-ancla o estructurante del grupo, concepto que facilita la identificación de la sinergia entre los proyectos. El proyectoancla debe poseer poder catalítico-sinérgico para justificar la formación de un agrupamiento en su entorno. No es necesariamente el proyecto de mayor porte, pues frecuentemente es un cuello de botella o eslabón faltante de la red de infraestructura, o de la cadena de valor productiva, o de la red educativa o de salud, que impide el aprovechamiento optimo de los efectos combinados del grupo, en beneficio del desarrollo económico, social y ambiental. El modelo elaborado (consensuado) se aplica al conjunto de proyectos necesarios para el desarrollo de cada JCI y macrorregión y permite identificar las prioridades y acciones estratégicas (SD-PCM, noviembre 2011: 8-10).

Se realizó una validación piloto de esta metodología multicriterio en distintos talleres con técnicos, profesionales y directivos de cada JCI: INTERNOR en Piura, los días 19, 20 Y 21 de setiembre de 2011; CENSUR, en Pasco, los días 3, 4, Y 5 de octubre; CIAM en Iquitos, los días 13, 14 Y 15 de octubre; y MACROSUR, en Moquegua, el 11 DE noviembre. Por último, se realizó un Taller de Consolidación a nivel nacional el día 5 de diciembre 2011.

Durante este proceso se priorizaron 28 proyectos estructurantes, los que se presentan a continuación:

JUNTA INTERREGIONAL DEL NORTE-INTERNOR (6): Proyecto agroindustrial del norte: Chavimochic, Chinecas, Alto Piura, Puyango Tumbes.; IIRSA Norte: Longitudinal Sierra, Paita, YurimaguasServicios logísticos-Puente aéreo; Fortalecimiento Portuario: Eje Paita, Bayóvar, Salaverry, Chimbote ; Circuitos Turísticos; Proyecto Hidro Energético del Marañón; Fortalecimiento de Ciencia y Tecnología en Universidades del norte.

JUNTA INTERREGIONAL DEL CENTRO SURCENSUR (8) :Carretera Huacho-Oyon-Ambo; Carretera Canta-Huayllay-Cerro de Pasco; Carretera Cañete-Yauyos-Chupaca-Junín ; Ferrocarril: Huánuco-Ucayali-Apurímac; Mega Puerto de Huacho; 
Aeropuerto Internacional Valle Mantaro:; Asfalto Carretera Villa Rica-Puerto Bermúdez-Pucallpa; Modernización Puerto de Marcona.

JUNTA INTERREGIONAL DE LA AMAZONÍACIAM (4) :Programa de Desarrollo Forestal Sostenible, Inclusivo y Competitivo de la Amazonía; Sistema Energético e Interconectividad; Programa de Desarrollo Alternativo, Integral e Inclusivo en Área de Influencia Cocalera; Cadena de Valor de cultivos alimenticios y agropecuarios.

JUNTA INTERREGIONAL DEL SUR - MACROSUR (10): Corredor vial Turístico y Productivo; Gasoducto y Petroquímica; Proyecto Integral de Camélidos Sudamericanos; Plataforma Logística del Sur; Programa de Afianzamiento de Cuencas; Programa de Minería con valor agregado; Programa de implementación de Energías Renovables; Megapuerto del Sur; Red ferroviaria del Sur; Programa de Saneamiento Básico.

\section{ANÁLISIS Y DISCUSIÓN. LAS JCI Y LA PROMOCIÓN DE LA INVERSIÓN DESCENTRALIZADA}

Un avance en el fortalecimiento de las JCI y los proyectos estructurantes fue el DU 058-2011, que establece normas excepcionales para agilizar las inversiones públicas ejecutadas por los Gobiernos regionales y locales, y que señala en su art. 13 que la SD-PCM desarrollarán un programa de desarrollo de capacidades en materia de gestión de inversiones en Gobiernos regionales y locales, Juntas de Coordinación Interregional y mancomunidades municipales, dando énfasis en proyectos de inversión articuladores en los espacios territoriales. Lamentablemente este programa no se implementó.

El papel asignado a las JCI, se refiere a la generación de propuestas estratégicas de desarrollo en los territorios asociados, gestionar proyectos conjuntos, formular planes de competitividad y promoción de la inversión, vigilar el cumplimiento de los planes de ordenamiento territorial y, en general, preparar condiciones y definir planes conducentes a una futura integración de los departamentos en una misma región (PNUD, 2009:18).

Los campos de intervención del Estado, tanto de regulación, de simplificación administrativa, de promoción o de servicios al inversionista, como de ejecución de inversión pública descentralizada, juegan un rol relevante en la atracción efectiva de la inversión privada, ya que su orientación es la de proveer servicios estatales e infraestructura pública necesaria para el bienestar social y el fomento de la inversión privada.
El artículo 8 de la Ley Marco de la Descentralización señala que los Gobiernos regionales y locales crearán las Agencias de Fomento de la Inversión Privada como un órgano especializado, consultivo y de coordinación con el sector privado, que se funda en acuerdos explícitos entre ambos sectores.

En la actualidad existen AFIP en varios gobiernos regionales (Piura, La Libertad, Amazonas, Madre de Dios, Ucayali, etc.), y en Juntas de Integración Interregional como CIAM, como la Agencia de Fomento de la Inversión Privada de la Amazonía-AMAZINV. Esta fue constituida en febrero de 2008. Conformada por cinco Gobiernos regionales (Loreto, Amazonas, Madre de Dios, San Martin y Ucayali), busca impulsar la inversión en la selva con proyectos vinculados a infraestructura, saneamiento, agroindustria, madera, negocios ambientales, acuicultura, turismo y biocomercio.

El Gobierno Regional de Piura ha reactivado la Agencia Regional de Fomento de la Inversión Privada, con el fin de impulsar la competitividad de Piura y su desarrollo sostenible. El Gobierno Regional de la Libertad, mediante la Agencia Regional de Fomento de la Inversión Privada ARFIP, formuló la Hoja de Ruta de Promoción de la Inversión Privada del Gobierno Regional de La Libertad para el periodo 2011 - 2016.

\section{CONCLUSIONES}

1. El objetivo nacional del proceso de descentralización es dinamizar la regionalización, mediante la conformación de macrorregiones transversales para reducir brechas territoriales y desigualdades sociales. Efraín Gonzales de Olarte $(2004,2011)$ ha señalado que nuestro país requiere de "regiones" con tamaño crítico, para que la descentralización sea sostenible en el largo plazo, gracias, entre otros factores, a la diversificación del sistema productivo, con productos/servicios de alto valor agregado, orientado al mercado nacional y para fines de exportación. En estas regiones, pueden establecerse círculos virtuosos: población electoral grande, crecimiento económico regional, base tributaria amplia y crecimiento fiscal, crecimiento económico, desarrollo regional basado en fuerzas endógenas y de inserción al mercado internacional.

2. En el caso de las Juntas de Coordinación Interregional, su objetivo es la gestión estratégica de integración, para la conformación de Regiones sostenibles, con acuerdos de articulación macrorregional. Las cinco Juntas 
de Coordinación Interregional agrupan a los 25 gobiernos regionales, a la Provincia Constitucional del Callao y a la Municipalidad Metropolitana de Lima.

3. Un aporte importante de la Secretaría de Descentralización de la PCM ha sido la aprobación de la Guía Metodológica para Priorización de Proyectos de Inversión y Articulación en Espacios Territoriales Descentralizados. El hecho de que las Juntas de Coordinación Interregional (mediante sus técnicos y autoridades) hayan identificado un conjunto de proyectos articuladores o estructurantes (28) es indicativo de la voluntad política de integración, así como la emergencia de potencialidades de desarrollo territorial. Para avanzar sería necesario hacer cambios en la política de inversión pública del MEF para financiamiento de proyectos interdepartamentales, y también introducir cambios en la normatividad de la descentralización para crear unidades ejecutoras en las JCI, de manera que puedan organizar una gestión interdepartamental, con agendas de desarrollo, unidades de planificación y programas de inversión multianuales con sus presupuestos.

\section{REFERENCIAS BIBLIOGRÁFICAS}

BARRIOS SUVELZA, Frank (2012). Estudio comparado de experiencias de descentralización relevantes para el Perú en la perspectiva de escenarios políticos de mediano plazo. PNUD/IDEA Internacional, La Paz, Bolivia.

GONZALES DE OLARTE, Efraín y ALEJOS, Walter (comp) .(2004). Departamentos o regiones: Lineamientos económicos y políticos y proyectos de ley para la integración y conformación de regiones. Lima.

GONZALES DE OLARTE, Efraín (2011). Descentralización, Divergencia y Desarrollo Regional en Opciones de Política Económica en el Perú, 2011-2015, PUCP, Lima.

PROGRAMA DE LAS NACIONES UNIDAS PARA EL DESARROLLO-PNUD (2006). Informe sobre Desarrollo Humano. Perú 2006. Hacia una Descentralización con Ciudadanía, Lima.

PROGRAMA DE LAS NACIONES UNIDAS PARA EL DESARROLLO-PNUD (2009). Informe de Desarrollo Humano-Perú 2009. Por una densidad del Estado al servicio de la gente, Lima.
Guía Metodológica para priorización de Proyectos de Inversión y Articulación en Espacios Territoriales Descentralizados. Secretaría de Descentralización, Lima noviembre.

PRESIDENCIA DEL CONSEJO DE MINISTROS, SECRETARÍA DE DESCENTRALIZACIÓN (2011). Seminario Internacional Descentralización y Territorio: Desafíos de la Ciudadania. Perú: Manuel Dammert: Un Enfoque Territorial de la Descentralización, Secretaría de Descentralización Lima, Diciembre.

PRESIDENCIA DEL CONSEJO DE MINISTROS, SECRETARÍA DE DESCENTRALIZACIÓN (2011). Propuesta de Plan Nacional de Descentralización y Regionalización (2012-2016). Documento para Consulta Nacional, Secretaría de Descentralización, Lima, diciembre.

PRESIDENCIA DEL CONSEJO DE MINISTROS. SECRETARIA DE DESCENTRALIZACION (2013). Informe Anual 2012 y Balance del proceso de descentralización 2002-2012, Secretaría de Descentralización, Lima, marzo.

Macroconsult.(2010). Consultoría para la formulación de una propuesta orientada a la efectivización del Plan Nacional de Regionalización. Secretaría de Descentralización de PCM, Lima.

MUÑOZ PORTUGAL, Ricardo (2013). Descentralización del desarrollo. Perspectivas del proceso peruano, Lima, abril.

TELLO, Mario (2008). Desarrollo económico local, descentralizacion y clusters: Teoría, evidencia y aplicaciones. CENTRUM Universidad Catolica, ,Lima.

SANTA CRUZ,Francisco (2007). La Regionalizacion y el fortalecimiento de las Juntas de Coordinacion Interregional. Cuadernos Descentralistas número 21 Grupo Propuesta Ciudadana. Lima, agosto.

USAID PERÚ DESCENTRALIZACIÓN (2011). Proceso de descentralizacion. Balance y agenda a julio de 2011. Lima, setiembre.

VERGARA, Ricardo. (2010). "Descentralizacion: una vez más ...ं..fracasamos?”. En: Perú Hoy: Centralismo y Concentración, DESCO, Lima. 